\title{
Non-linear Age-dependent Population Dynamics
}

\author{
Morton E. Gurtin \& Richard C. MacCamy
}

\section{Introduction}

The simplest model of population dynamics is based on the Malthusian law

$$
\dot{P}=\delta P \quad(\delta=\text { constant }),
$$

where $P(t)$ is the total population at time $t$ and $\delta$ is the growth modulus. This law is clearly inapplicable to situations in which the population competes for resources (e.g., space and food), for in these situations $\delta$ should depend on the size of the population: the larger the population, the slower should be its rate of growth.

To overcome this deficiency in the Malthusian law, VERHULST $[1845,1847]$ assumed that

$$
\dot{P}=\left(\delta_{0}-\omega_{0} P\right) P \quad\left(\delta_{0}, \omega_{0}=\text { constant }\right) .
$$

For $\delta_{0}$ and $\omega_{0}$ positive this differential equation has a stable equilibrium point $P=\xi=\delta_{0} / \omega_{0}$, and populations with $P(0)<\xi$ grow monotonically to $\xi$ as $t \rightarrow \infty$. The solution of (1.2),

$$
P(t)=\frac{\xi}{1+\left(\frac{\xi}{P(0)}-1\right) e^{-\delta_{0} t}},
$$

has been applied, with remarkable success, to fit the growth curves of various types of populations. ${ }^{1}$

The chief disadvantage of the Malthus and Verhulst models is that they yield no information whatsoever concerning the age distribution of the population, and, in fact, are based on the tacit assumption that the birth and death processes are age-independent. A model applicable to age-dependent population dynamics was first proposed by LOTKA and von FoERSTER. ${ }^{2}$ This model is based on the assumption that

$$
P(t)=\int_{0}^{\infty} \rho(a, t) d a
$$

\footnotetext{
1 See, e.g., LoTKA [1925], pp. 66-76.

2 The basic ideas behind this model are contained in the work of LoTKA [1925], where (1.7) and (1.8) are implicit. The partial differential equation (1.6) is due to voN FoERSTER [1959], although with $d=0$ it appears earlier in the work of SCHERBAUM \& RASCH [1957]. See also Fisher [1958], Lopez [1961], Trucco [1965], Keyfitz [1968], Crow \& Kimura [1970], LANGHAAR [1972].
} 
where $\rho(a, t)$ is the population at time $t$ in the age-interval $(a, a+d a)$. Consider the group of individuals who are of age $a$ at time $t$. If $t$ is increased by $h$ units, these individuals age by $h$ units; thus

$$
D \rho(a, t)=\lim _{h \rightarrow 0} \frac{\rho(a+h, t+h)-\rho(a, t)}{h}
$$

is the rate at which the population of this group is changing in time. This rate plus the number $d(a, t)$ of individuals (per unit age and time) of age $a$ who die at time $t$ must equal zero:

$$
D \rho+d=0 \text {. }
$$

One then assumes that

$$
d(a, t)=\lambda(a) \rho(a, t),
$$

and that the birth process is described by the "renewal equation"

$$
\rho(0, t)=\int_{0}^{\infty} \beta(a) \rho(a, t) d a .
$$

Here $\beta(a)$, called the birth-modulus, is the average number of offsprings (per unit time) produced by an individual of age $a ; \lambda(a)$, called the death-modulus, is the death-rate at age $a$ per unit population of age $a .^{1}$ The system (1.6)-(1.8), supplemented by the initial condition

$$
\rho(a, 0)=\varphi(a)
$$

constitute the Lotka-von Foerster model. As we shall see later, $\rho$ will generally not be differentiable everywhere; of course, when it is,

$$
D \rho=\rho_{a}+\rho_{t} .
$$

The same objection can be raised to the Lotka-von Foerster model as was raised previously to the Malthusian law: the birth and death moduli are independent of the population $P$. To rectify this we allow $\beta$ and $\lambda$ to depend on $P$. Our theory is therefore based on the following system of equations:

$$
\begin{gathered}
D \rho+\lambda(a, P) \rho=0, \\
P(t)=\int_{0}^{\infty} \rho(a, t) d a, \\
\rho(0, t)=\int_{0}^{\infty} \beta(a, P) \rho(a, t) d a,
\end{gathered}
$$

supplemented, of course, by the initial condition (1.9).

The problem (1.9), (1.11) is studied in detail in Section 2. There we lay down our basic hypotheses: that $\lambda, \beta$, and $\varphi$ be non-negative and sufficiently smooth

\footnotetext{
${ }^{1}$ See, e.g., ANDREWARTHA \& BIRCH [1954], where curves of $\beta(a)$ and
}

$$
\pi(a)=\exp \left(-\int_{0}^{\infty} \lambda(\alpha) d \alpha\right)
$$

are given for the vole mouse (microtus agrestis) and for the rice weevil (calandra oryzae). 
(in a certain precise sense). We then establish the existence of a unique solution on a sufficiently small time-interval, and we prove that if (in addition to the above hypotheses) $\beta$ is uniformly bounded, then existence and uniqueness hold for all time. We show further, by example, that some such additional hypothesis is necessary for global existence. Our solutions are all in the sense of $(1.11)_{1}$ : $\rho$ is not required to possess partial derivatives with respect to $a$ and $t$, but only the directional derivative (1.5). We discuss conditions which guarantee that the solution actually be of class $C^{1}$; these turn out to be compatibility conditions on the initial data $\varphi$.

All of our results are based on the reduction of the problem (1.9), (1.11) to a pair of non-linear functional equations for the total population $P(t)$ and the birth-rate $B(t)=\rho(0, t)$. These equations are natural extensions of the linear Volterra integral equation for $B(t)$ which occurs in the Lotka-von Foerster theory. ${ }^{1}$ We study these functional equations by means of a fixed point argument.

In Section 3 we study equilibrium age distributions; that is, solutions $\rho$ of (1.11) which are independent of time. We show that in our non-linear theory a greater variety of such solutions is available than in the linear theory of LoTKA and VON FOERSTER. We also study the stability of these equilibrium age distributions. In particular, we give conditions under which such distributions are exponentially asymtotically stable.

Section 4 contains a discussion of some special cases, corresponding to particular choices of $\lambda$ and $\beta$, in which our model reduces to a pair of non-linear ordinary differential equations for $P(t)$ and $B(t)$. In particular, we show that the models of Malthus and VerHulst can be obtained in this manner. We emphasize, however, that even in these simple cases our study provides new information: it enables us to determine the evolution in time of an initial age distribution $\rho(a, 0)$.

The theory described above can clearly be extended to include several interacting species, and we intend to pursue this problem in the near future. A second interesting generalization of our theory occurs when we take spatial diffusion into account. ${ }^{2}$ The analogs of our present theory in the diffusion problem present several very challenging questions, and these too we hope to pursue.

\section{Existence and Uniqueness}

In this section we shall establish an alternative formulation of the population problem (1.9), (1.11) in terms of a pair of coupled integral equations for the birth-rate $B(t)$ and the total population $P(t)$, and we shall use these equations to prove existence and uniqueness. It is clear that our problem is physically meaningful only if $\varphi(a), \lambda(a, P)$, and $\beta(a, P)$ are non-negative. Also, since we want the initial total population to be finite, $\varphi$ should belong to $L_{1}\left(\mathbb{R}^{+}\right)$. We collect these assumptions, together with some others of a more technical nature, in the following hypotheses: ${ }^{3}$

\footnotetext{
${ }^{1}$ See, e.g., KeYfitz [1968], Eq. (5.1.1).

2 Simple linear models for diffusion of a single species have already been proposed by SKELLAM [1951], KERNER [1959], and GURTIN [1973].

${ }^{3}$ We use the following notation throughout: $\mathbb{R}=(-\infty, \infty) ; \mathbb{R}^{+}=[0, \infty) ; C(A: B)$ is the set of all continuous functions from $A$ to $B ; C(A)=C(A: \mathbb{R})$.
} 
$\left(\mathrm{H}_{1}\right) \varphi \in L_{1}\left(\mathbb{R}^{+}\right)$is piecewise continuous;

$\left(\mathrm{H}_{2}\right) \quad \lambda, \beta \in C\left(\mathbb{R}^{+} \times \mathbb{R}^{+}\right) ; \lambda_{P}(a, P)$ and $\beta_{P}(a, P)$ exist for all $a \geqq 0$ and $P \geqq 0 ; \lambda(\cdot, P), \lambda_{P}(\cdot, P), \beta(\cdot, P)$, and $\beta_{P}(\cdot, P)$, as functions of $P$, belong to $C\left(\mathbb{R}^{+}: L_{\infty}\left(\mathbb{R}^{+}\right)\right)$;

$\left(\mathrm{H}_{3}\right) \varphi \geqq 0, \lambda \geqq 0, \beta \geqq 0$.

For convenience, we assume, once and for all, that $\left(\mathrm{H}_{1}\right)-\left(\mathrm{H}_{3}\right)$ are satisfied.

By a solution of the population problem up to time $T>0$ we mean a non-negative function $\rho$ on $\mathbb{R}^{+} \times[0, T]$ with the following properties: $D \rho$ exists on $\mathbb{R}^{+} \times[0, T]$; $\rho(\cdot, t) \in L_{1}\left(\mathbb{R}^{+}\right)$and

is continuous for $0 \leqq t \leqq T$;

$$
P(t)=\int_{0}^{\infty} \rho(a, t) d a
$$

$$
\begin{array}{ll}
D \rho(a, t)+\lambda(a, P(t)) \rho(a, t)=0 & (a>0,0<t<T), \\
\rho(0, t)=\int_{0}^{\infty} \beta(a, P(t)) \rho(a, t) d a & (0<t \leqq T), \\
\rho(a, 0)=\varphi(a) & (a \geqq 0) .
\end{array}
$$

It is important to note that $(2.2)_{2}$ is not required to hold at $t=0$. Indeed, by $(2.2)_{3}$ this relation will be satisfied at $t=0$ if and only if $\varphi$ satisfies the compatibility condition

$$
\varphi(0)=\int_{0}^{\infty} \beta(a, \Phi) \varphi(a) d a, \quad \Phi=\int_{0}^{\infty} \varphi(a) d a,
$$

which is simply the requirement that the initial data be consistent with the birth process. We do not impose the restriction (2.3), because we envisage situations in which the initial age distribution $\varphi$ is completely arbitrary.

Let $\rho$ be a solution up to time $T$, let $\left(a_{0}, t_{0}\right) \in \mathbb{R}^{+} \times[0, T]$, and let

$$
\bar{\rho}(h)=\rho\left(a_{0}+h, t_{0}+h\right), \quad \bar{\lambda}(h)=\lambda\left(a_{0}+h, P\left(t_{0}+h\right)\right) .
$$

Then (1.5) and (2.2) imply that

$$
\frac{d \bar{\rho}}{d h}+\bar{\lambda}(h) \bar{\rho}=0
$$

and this equation has the unique solution

$$
\rho\left(a_{0}+h, t_{0}+h\right)=\rho\left(a_{0}, t_{0}\right) e^{-\int_{0}^{h} \bar{\lambda}(\eta) d \eta},
$$

giving the values of $\rho$ at all points on the characteristic through $\left(a_{0}, t_{0}\right)$ in terms of the value of $\rho$ at $\left(a_{0}, t_{0}\right)$. In particular, if in (2.6) we take $\left(a_{0}, t_{0}\right)=(a-t, 0)$ and $h=t$, we conclude, with the aid of $(2.2)_{3}$, that

$$
\rho(a, t)=\varphi(a-t) e^{-\int_{0}^{t} \lambda(a-t+t, P(\tau)) d \tau} \quad \text { for } a \geqq t .
$$


On the other hand, the substitutions $\left(a_{0}, t_{0}\right)=(0, t-a)$ and $h=a$ in (2.6) lead to the conclusion that

where

$$
\rho(a, t)=B(t-a) e^{-\int_{0}^{a} \lambda(\alpha, P(t-a+\alpha)) d \alpha} \quad \text { for } t>a,
$$

$$
B(t)=\rho(0, t)
$$

is the birth-rate. Finally, if we substitute (2.7) and (2.8) into (2.1) and $(2.2)_{2}$, we arrive at the following pair of coupled integral equations for $P$ and $B$ :

where

$$
\begin{aligned}
P(t)= & \int_{0}^{t} K(t-a, t ; P) B(a) d a+\int_{0}^{\infty} L(a, t ; P) \varphi(a) d a, \\
B(t)= & \int_{0}^{t} \beta(t-a, P(t)) K(t-a, t ; P) B(a) d a \\
& +\int_{0}^{\infty} \beta(a+t, P(t)) L(a, t ; P) \varphi(a) d a,
\end{aligned}
$$

$$
\begin{aligned}
& K(\alpha, t ; P)=e^{-\int_{t-\alpha}^{t} \lambda(\alpha+\tau-t, P(\tau)) d \tau} \\
& L(\alpha, t ; P)=e^{-\int_{0}^{t} \lambda(\tau+\alpha, P(\tau)) d \tau} .
\end{aligned}
$$

The integral equations (2.10) form the basis for the discussion in this section. Note that $K$ and $L$ are functionals of $P$. When $\lambda$ and $\beta$ are independent of $P$, $(2.10)_{2}$ is the classical linear integral equation of LoTKA ${ }^{1}$ for the birth-rate $B$, while $(2.10)_{1}$ is simply a formula for $P$.

From (2.7) it follows that discontinuities in $\varphi$ propagate along characteristics. Further, even when $\varphi$ is continuous, (2.7) and (2.8) imply that $\rho$ will be discontinuous across the characteristic $t=a$ unless $B\left(0^{+}\right)=\varphi(0)$. By $(2.10)_{2}, B\left(0^{+}\right)$ is equal to the right-hand side of (2.3). Thus (when $\varphi$ is continuous) a necessary and sufficient condition that $\rho$ be continuous across $t=a$ is that (2.3) hold. When (2.3) is not satisfied, $B$, defined by (2.9), will exhibit a discontinuity at $t=0$, and therefore $B$ defined at $t=0$ by (2.9) will not agree with $B$ defined at $t=0$ by $(2.10)_{2}$. To avoid this (technical) difficulty we define $B$ by $(2.9)$ for $t>0$ and take $B(0)=$ $B\left(0^{+}\right)$.

Theorem 1. Let $\rho$ be a solution of the population problem up to time $T>0$. Then the total population $P$ and the birth-rate $B$ satisfy the integral equations (2.10) on $[0, T]$. Conversely, if $P$ and $B$ are non-negative continuous functions that satisfy (2.10) on $[0, T]$, and if $\rho$ is defined on $\mathbb{R}^{+} \times[0, T]$ by $(2.7),(2.8)$, then $\rho$ is a solution of the population problem up to time $T$.

Proof. We have already established the first portion of the theorem. To prove the converse assertion, let $P \geqq 0$ and $B \geqq 0$ be continuous functions on $[0, T]$ consistent with (2.10), and let $\rho$ be defined on $\mathbb{R}^{+} \times[0, T]$ by $(2.7),(2.8)$. Then $\rho \geqq 0$ (since $\varphi$ and $\beta$ are non-negative), $(2.3)_{3}$ holds, $\rho(0, t)=B(t)$ for $t>0$, and $\rho(\cdot, t) \in L_{1}\left(\mathbb{R}^{+}\right)$(since $\lambda, B$, and $P$ are continuous and $\varphi \in L_{1}\left(\mathbb{R}^{+}\right)$). It there-

1 See, e.g., KeYfitz [1968], Eq. (5.1.1). 
fore follows from $(2.7),(2.8),(2.10)$, and (2.11) that (2.1) and $(2.2)_{2}$ hold. Finally, (1.5), (2.6), and (2.7) imply that $D \rho$ exists on $\mathbb{R}^{+} \times[0, T]$ and that $(2.2)_{1}$ is satisfied. This completes the proof.

Theorem 2. There exists a $T>0$ such that the population problem has a unique solution up to time $T$.

Proof. Let

$$
C^{+}[0, T]=\{f \in C[0, T] \mid f \geqq 0\} .
$$

In view of Theorem 1 it suffices to find functions $P, B \in C^{+}[0, T]$ that satisfy the integral equations (2.10). We shall solve these equations in the following manner. Consider first $(2.10)_{2}$. For fixed $P \in C^{+}[0, T]$ this is a linear Volterra integral equation for $B$, and hence it has a unique solution on $[0, T]$ which we denote by

$$
B(t)=\mathscr{B}_{T}(P)(t)
$$

Using this solution, we can define an operator $\mathscr{P}_{T}$ on $C^{+}[0, T]$ by the right-hand side of $(2.10)_{1}$ with $B$ replaced by $\mathscr{B}_{T}(P)$ :

$$
\mathscr{P}_{T}(P)(t)=\int_{0}^{t} K(a-t, t ; P) \mathscr{B}_{T}(P)(a) d a+\int_{0}^{\infty} L(a, t ; P) \varphi(a) d a .
$$

Clearly, our hypotheses $\left(\mathrm{H}_{1}\right)-\left(\mathrm{H}_{3}\right)$ imply that

$$
\mathscr{B}_{T}, \mathscr{P}_{T}: C^{+}[0, T] \rightarrow C^{+}[0, T] \text {. }
$$

Thus the population problem reduces to finding a fixed point $P$ of the operator $\mathscr{P}_{T}$, and hence Theorem 2 is a direct consequence of the following:

Lemma. There exists a $T>0$ such that the operator $\mathscr{P}_{T}: C^{+}[0, T] \rightarrow C^{+}[0, T]$ defined by (2.14) has a unique fixed point.

We postpone, until Section 4, our proof of this lemma. We remark here, however, that our proof, in principle, is constructive, since it is based on the contractive mapping theorem. Indeed, one starts with $P^{0}$ equal to the constant function $\Phi=\int_{0}^{\infty} \varphi(a) d a$, and then iterates by taking $P^{n+1}=\mathscr{P}\left(P^{n}\right)$. This method, however, requires the a priori solution of $(2.10)_{2}$ at each step. One is tempted to use the seemingly more straightforward procedure of iterating on both of (2.10) jointly. Here one should start with $P^{0}=\Phi$ and $B^{0}=\int_{0}^{\infty} \beta(a, \Phi) \varphi(a) d a$ and then iterate by taking $P^{n+1}$ and $B^{n+1}$ as the left-hand side of (2.10) with $P^{n}$ and $B^{n}$ substituted on the right-hand side. We were able to prove convergence of this process only for those situations in which the initial total population $\Phi$ is sufficiently small. In contrast, our process is convergent for arbitrary $\Phi$.

Theorem 2 is a local existence theorem. Indeed, as is clear from the last example of Section 4, this is all that can be expected in the absence of any further hypotheses concerning $\beta$ or $\lambda$. What is needed to obtain global existence is some type of a priori estimate. With this in mind we now consider $\beta$ and $\lambda$ more carefully. Recall that $\beta(a, P)$ is the average number of offsprings (per unit time) 
produced by an individual of age $a$ when the total population is $P$. It therefore seems reasonable to expect that this quantity will be uniformly bounded for all $a$ and $P$; that is,

On the other hand, by $\left(\mathrm{H}_{3}\right)$

$$
\bar{\beta}=\sup _{\substack{a \geq 0 \\ P \geqq 0}} \beta(a, P)<\infty .
$$

$$
\lambda=\inf _{\substack{a \geq 0 \\ P \geqq 0}} \lambda(a, P)
$$

is not only finite but non-negative. When (2.16) is satisfied we call

$$
\delta=\vec{\beta}-\underline{\lambda}
$$

the bounding growth rate. This terminology is justified by our next result, which shows that $P$ and $B$ can grow at most like $e^{\delta t}$; that is, at most like a Malthusian population with growth rate $\delta$.

Theorem 3. Assume that (2.16) holds. Let $\rho$ be a solution of the population problem up to time $T$. Then for $0 \leqq t \leqq T$

and

$$
\begin{aligned}
& P(t) \leqq \Phi e^{\delta t}, \\
& B(t) \leqq \beta \Phi e^{\delta t},
\end{aligned}
$$

$$
\rho(a, t) \leqq \bar{\beta} \Phi e^{-\underline{\lambda} a} e^{\delta t} \quad(a<t), \quad \rho(a, t) \leqq\|\varphi\|_{t} e^{-\underline{\lambda} a} \quad(a \geqq t),
$$

where $\Phi$ is the initial total population $(2.3)_{2}$, while $\|\varphi\|_{t}=\sup _{[0, t]} \varphi$.

Proof. From (2.11)

$$
K(\alpha, t ; P) \leqq e^{-\underline{\lambda} \alpha}, \quad L(\alpha, t ; P) \leqq e^{-\underline{\lambda} t} .
$$

Thus $(2.10)_{2}$ and (2.16) yield

$$
B(t) \leqq \bar{\beta} \int_{0}^{t} e^{-\underline{\lambda}(t-a)} B(a) d a+\bar{\beta} \Phi e^{-\underline{\lambda} t},
$$

and, by virtue of Gronwall's inequality, this clearly implies (2.19) $)_{2}$. If we substitute this result into $(2.10)_{1}$ and use (2.21), we are led, at once, to $(2.19)_{1}$. Finally, (2.20) is an obvious consequence of (2.7), (2.8), and (2.19).

Note that if $\varphi$ is uniformly bounded, so that $\varphi_{\max }=\sup \varphi<\infty$, then (2.20) implies that

$$
\rho(a, t) \leqq C e^{\delta t}
$$

for $a \geqq 0$ and $0 \leqq t \leqq T$, where $C$ is the largest of $\varphi_{\max }, \bar{\beta} \Phi$.

As we shall now see, assumption (2.16) leads, via the a priori estimate $(2.19)_{1}$, to global existence.

Theorem 4. Assume that (2.16) holds. Then the population problem has a unique solution for all time.

The proof of this theorem will be given in Section 5. In Section 4 we shall give an example in which (2.16) is not satisfied and for which $P(t) \rightarrow \infty$ in a finite time. 
As we have mentioned previously, a necessary and sufficient condition for $\rho$ to be continuous is that $\varphi$ be continuous and satisfy the compatibility condition (2.3). The next theorem, the proof of which we postpone until Section 5, gives an additional compatibility condition that must be satisfied if $\rho$ is to be of class $C^{1}$.

Theorem 5. Assume that $\varphi \in C^{1}\left(\mathbb{R}^{+}\right)$with $\dot{\varphi} \in L_{1}\left(\mathbb{R}^{+}\right)$. Assume, in addition, that $\lambda, \beta \in C^{1}\left(\mathbb{R}^{+} \times \mathbb{R}^{+}\right)$and that the mappings carrying $(t, P)$ into the functions $a \mapsto \beta_{a}(a+t, P)$ and $a \mapsto \beta_{P}(a+t, P)$ belong to $C\left(\mathbb{R}^{+} \times \mathbb{R}^{+}: L_{\infty}\left(\mathbb{R}^{+}\right)\right)$. Let $\rho$ be a solution of the population problem up to time $T$. Then $\rho \in C^{1}\left(\mathbb{R}^{+} \times[0, T]\right)$ if and only if $\varphi$ satisfies the compatibility conditions (2.3) and

$$
\begin{aligned}
\dot{\varphi}(0)= & {[\lambda(0, \Phi)-\beta(0, \Phi)] \varphi(0) } \\
& -\int_{0}^{\infty}\left[\beta_{a}(a, \Phi)+\beta_{p}(a, \Phi) \dot{\Phi}-\beta(a, \Phi) \lambda(a, \Phi)\right] \varphi(a) d a,
\end{aligned}
$$

where

$$
\dot{\Phi}=\varphi(0)-\int_{0}^{\infty} \lambda(a, \Phi) \varphi(a) d a
$$

\section{Equilibrium Age Distributions. Stability}

In this section we study solutions of (1.12) in which $\rho(a, t) \equiv \rho(a)$ is independent of the time $t$. Clearly, a density field of this type will satisfy $(2.2)_{1,2}$ if and only if

$$
\begin{aligned}
\rho_{a}+\lambda(a, P) \rho=0, & \\
P & =\int_{0}^{\infty} \rho(a) d a, \\
\rho(0) & =\int_{0}^{\infty} \beta(a, P) \rho(a) d a .
\end{aligned}
$$

It is important to note that in this case both $P$ and the birth-rate $B=\rho(0)$ are constants. A solution $\rho \in C^{1}\left(\mathbb{R}^{+}\right)$of (3.1) will be referred to as an equilibrium age distribution. In the study of such age distributions the following quantities are of prime importance:

$$
\pi(a, P)=e^{-\int_{0}^{a} \lambda(\alpha, P) d \alpha},
$$

the probability that a person will survive to age $a$ if the population is held constant at $P$, and

$$
R(P)=\int_{0}^{\infty} \beta(a, P) \pi(a, P) d a,
$$

the number of children expected to be born to an individual when the population is $P$.

Theorem 6. ${ }^{1}$ Let $P>0$ and assume that $\beta(\cdot, P) \pi(\cdot, P) \in L_{1}\left(\mathbb{R}^{+}\right)$. Then a necessary and sufficient condition that an equilibrium age distribution exist with

\footnotetext{
${ }^{1}$ As is clear from the proof, hypotheses $\left(\mathrm{H}_{1}\right)-\left(\mathrm{H}_{3}\right)$ are not needed for the validity of these theorems.
} 
total population $P$ is that

$$
R(P)=1 .
$$

When this is the case the (unique) equilibrium age distribution corresponding to $P$ is given by

with

$$
\rho(a)=B \pi(a, P)
$$

$$
B=\frac{P}{\int_{0}^{\infty} \pi(a, P) d a} .
$$

Proof. The unique solution of $(3.1)_{1,2}$ (with initial condition $\rho(0)=B$ ) is (3.5), (3.6). Thus to complete the proof it suffices to show that, granted (3.5), (3.4) is equivalent to $(3.1)_{3}$. But this is obvious.

In the classical (linear) theory $R$ is independent of $P$, and it would be fortuitous for $R$ to equal one; here, however, $R(P)$ is a function of $P$, and in most problems of interest there will exist at least one value of $P$ for which $R(P)=1$.

Our next step is to study the stability of a given equilibrium age distribution $\rho_{0}(a)$ with birth-rate $B_{0}$ and total population $P_{0}$. Thus we consider "perturbations" $\xi(a, t)$ of $\rho_{0}(a)$ and write

$$
\begin{gathered}
\rho(a, t)=\rho_{0}(a)+\xi(a, t), \\
P(t)=P_{0}+p(t) .
\end{gathered}
$$

A simple computation then shows that $\rho$ and $P$ obey the basic equations (2.1) and (2.2) 1,2 if and only if $\xi$ and $p$ satisfy

$$
\begin{aligned}
D \xi(a, t) & +\lambda_{0}(a) \xi(a, t)+\omega(a) p(t)=\infty(a, t), \\
p(t) & =\int_{0}^{\infty} \xi(a, t) d a, \\
\xi(0, t) & =\int_{0}^{\infty} \beta_{0}(a) \xi(a, t) d a+\kappa p(t)+\psi(t),
\end{aligned}
$$

where

$$
\begin{gathered}
\lambda_{0}(a)=\lambda\left(a, P_{0}\right), \quad \beta_{0}(a)=\beta\left(a, P_{0}\right), \quad \pi_{0}(a)=\pi\left(a, P_{0}\right), \\
\lambda_{0}^{\prime}(a)=\lambda_{P}\left(a, P_{0}\right), \quad \beta_{0}^{\prime}(a)=\beta_{P}\left(a, P_{0}\right), \quad \omega(a)=B_{0} \lambda_{0}^{\prime}(a) \pi_{0}(a), \\
\kappa=B_{0} \int_{0}^{\infty} \beta_{0}^{\prime}(a) \pi_{0}(a) d a, \\
x(a, t)=-\lambda_{0}^{\prime}(a) p(t) \xi(a, t)-\Lambda(a, p(t))\left[B_{0} \pi_{0}(a)+\xi(a, t)\right], \\
\psi(t)=p(t) \int_{0}^{\infty} \beta_{0}^{\prime}(a) \xi(a, t) d a+\int_{0}^{\infty} \Omega(a, p(t))\left[B_{0} \pi_{0}(a)+\xi(a, t)\right] d a, \\
\Lambda(a, p)=\lambda\left(a, P_{0}+p\right)-\lambda_{0}(a)-\lambda_{0}^{\prime}(a) p, \\
\Omega(a, p)=\beta\left(a, P_{0}+p\right)-\beta_{0}(a)-\beta_{0}^{\prime}(a) p .
\end{gathered}
$$


In the presence of sufficient smoothness, the terms $x$ and $\psi$ are of "secondorder" in the quantities $\xi$ and $p$. It therefore seems reasonable to expect that the linear equations which result when these terms are neglected would determine the behavior of the population in a neighborhood of the equilibrium distribution $\rho_{0}$. With this in mind, we now consider the linear system

$$
\begin{aligned}
D \xi & +\lambda_{0} \xi+\omega p=0, \\
p(t) & =\int_{0}^{\infty} \xi(a, t) d a, \\
\xi(0, t) & =\int_{0}^{\infty} \beta_{0}(a) \xi(a, t) d a+\kappa p(t),
\end{aligned}
$$

which represents an interesting generalization of the classical linear Lotka-von Foerster equations.

To study the stability of the equilibrium distribution $\rho_{0}$, we consider solutions of (3.10) of the form

$$
\xi(a, t)=\tilde{\xi}(a) e^{\gamma t}
$$

with $\tilde{\xi}(a)$ and $\gamma$ complex. Clearly, $\xi$ satisfies (3.10) if and only if

$$
\begin{aligned}
\tilde{\xi}_{a}+\left(\lambda_{0}+\gamma\right) \tilde{\xi}+\omega \tilde{p}=0, \\
\tilde{p}=\int_{0}^{\infty} \tilde{\xi}(a) d a, \\
\tilde{\xi}(0)=\int_{0}^{\infty} \beta_{0}(a) \tilde{\xi}(a) d a+\tilde{\kappa} p .
\end{aligned}
$$

It is a simple matter to verify that $(3.12)_{1,2}$ are equivalent to the equation

where

$$
\tilde{\xi}(a)=\tilde{\xi}(0) \pi_{0}(a)\left[e^{-\gamma a}-g_{\gamma} f_{\gamma}(a)\right],
$$

$$
f_{\gamma}(a)=\int_{0}^{a} e^{-\gamma(a-\alpha)} \lambda_{0}^{\prime}(\alpha) d \alpha, \quad g_{\gamma}=\frac{B_{0} \int_{0}^{\infty} e^{-\gamma a} \pi_{0}(a) d a}{1+B_{0} \int_{0}^{\infty} \pi_{0}(a) f_{\gamma}(a) d a} .
$$

Further, (3.12) $)_{3}$ will be satisfied if and only if

$$
1=\int_{0}^{\infty} r(a) e^{-\gamma a} d a+g_{\gamma}\left[\frac{\kappa}{B_{0}}-\int_{0}^{\infty} r(a) f_{\gamma}(a) d a\right], \quad r=\pi_{0} \beta_{0} .
$$

Thus a solution of the form (3.11) will exist if and only if $\gamma$ satisfies the transcendental equation (3.15). If all $\gamma$ 's that satisfy (3.17) have negative real parts, then all solutions of the form (3.11) will tend to zero as $t$ tends to infinity. This is a standard test for the stability of an equilibrium point. Our next theorem shows that in the present situation this is, indeed, a valid test.

In addition to $\left(\mathrm{H}_{1}\right)-\left(\mathrm{H}_{3}\right)$, we need the following hypotheses concerning the equilibrium distribution $\rho_{0}$ : 
$\left(\mathrm{H}_{4}\right) \frac{\partial^{k}}{\partial a^{k}} \lambda\left(a, P_{0}\right)$ and $\frac{\partial^{k}}{\partial a^{k}} \beta\left(a, P_{0}\right)$ exist for $k=1,2$, and, as functions of $a$, belong to $L_{\infty}\left(\mathbb{R}^{+}\right)$.

$\left(\mathrm{H}_{5}\right) \frac{\Lambda(a, p)}{p}$ and $\frac{\Omega(a, p)}{p}$ tend to zero as $p \rightarrow 0$ uniformly for $a \rightarrow 0$.

$\left(\mathrm{H}_{6}\right) \lambda^{*}=\inf _{a \geqq 0} \lambda\left(a, P_{0}\right)>0$.

Theorem 7. Assume that (3.15) has no solution $\gamma$ with $\operatorname{Re}(\gamma) \geqq 0$. Then there exist numbers $\delta>0$ and $\mu>0$ such that given any initial data $\varphi$ with $\left\|\varphi-\rho_{0}\right\|_{L_{1}}<\delta$, the corresponding solution of the population problem, if it exists for all time, satisfies

$$
\begin{aligned}
P(t)-P_{0} & =O\left(e^{-\mu t}\right) \\
\rho(a, t)-\rho_{0}(a) & =O\left(e^{-\mu t}\right) \quad(\text { for each } a)
\end{aligned}
$$

as $t \rightarrow \infty$.

\section{Some Simple Examples}

Our theory is greatly simplified if we assume that

$$
\lambda(a, P)=\lambda(P), \quad \beta(a, P)=\beta(P) e^{-\alpha a}
$$

for all $a$ and $P$, with

$$
\lambda(P)>0, \quad \beta(P)>0, \quad \alpha \geqq 0 .
$$

When this is the case (3.2) and (3.3) imply that

$$
\begin{aligned}
\pi(a, P) & =e^{-\lambda(P) a}, \\
R(P) & =\frac{\beta(P)}{\lambda(P)+\alpha},
\end{aligned}
$$

and we conclude from the first theorem in Section 3 that there exists an equilibrium age distribution $\rho_{0}(a)$ with total population $P_{0} \neq 0$ if and only if

in which case

$$
\beta\left(P_{0}\right)=\lambda\left(P_{0}\right)+\alpha
$$

$$
\begin{aligned}
\rho_{0}(a) & =\lambda\left(P_{0}\right) P_{0} e^{-\lambda\left(P_{0}\right) a}, \\
B_{0} & =\lambda\left(P_{0}\right) P_{0} .
\end{aligned}
$$

Here, of course, $B_{0}=\rho_{0}(0)$ is the equilibrium birth-rate.

To investigate the stability of the equilibrium distribution $\rho_{0}$ we could study the transcendental equation (3.17). However it is far more instructive to postpone until later our discussion of stability and to study now the form our basic equations take in the present circumstances.

In view of the assumptions (4.1), equations (2.11) imply that

$$
\begin{aligned}
\frac{\partial}{\partial t} K(t-a, t ; P) & =-\lambda(P(t)) K(t-a, t ; P), \\
\frac{\partial}{\partial t} L(a, t ; P) & =-\lambda(P(t)) L(a, t ; P) .
\end{aligned}
$$


In addition,

$$
\frac{\partial}{\partial t} \beta(t \pm a, P(t))=\frac{\beta^{\prime}(P(t))}{\beta(P(t))} \dot{P}(t) \beta(t \pm a, P(t))-\alpha \beta(t \pm a, P(t)) .
$$

If we differentiate (2.10) with respect to $t$, we conclude, with the aid of (4.6) and (4.7), that

$$
\begin{array}{r}
\dot{P}+\lambda(P) P-B=0 \\
\dot{B}-\frac{\beta^{\prime}(P)}{\beta(P)} B \dot{P}+[\lambda(P)+\alpha-\beta(P)] B=0 .
\end{array}
$$

On the other hand, if we let $t \rightarrow 0$ in (2.10) we arrive at

$$
P(0)=\int_{0}^{\infty} \varphi(a) d a, \quad B(0)=\beta(P(0)) \int_{0}^{\infty} e^{-\alpha a} \varphi(a) d a .
$$

Thus our problem reduces to finding a solution of the differential equations (4.8) subject to the initial conditions (4.9). Of course, once (4.8), (4.9) is solved, $\rho$ is computed using (2.6) and (2.7).

Note that, as would be expected, the (non-trivial) equilibrium points of (4.8) occur when $P$ and $B$ have values consistent with (4.4) and (4.5) 2 . To investigate the stability of such an equilibrium point $\left(P_{0}, B_{0}\right)$, we let

$$
P=P_{0}+p, \quad B=B_{0}+b
$$

and linearize (4.8) with respect to $p$ and $b$ in a neighborhood of $\left(P_{0}, B_{0}\right)$; the result is

where

$$
\begin{aligned}
\dot{p}+A_{1} p-b & =0, \\
\dot{b}+A_{2} \dot{p}+A_{3} p & =0
\end{aligned}
$$

$$
A_{1}=\lambda\left(P_{0}\right)+\lambda^{\prime}\left(P_{0}\right) P_{0}, \quad A_{2}=-B_{0} \frac{\beta^{\prime}\left(P_{0}\right)}{\beta\left(P_{0}\right)}, \quad A_{3}=B_{0}\left[\lambda^{\prime}\left(P_{0}\right)-\beta^{\prime}\left(P_{0}\right)\right] .
$$

Combining these equations we find that

$$
\ddot{p}+\left(A_{1}+A_{2}\right) \dot{p}+A_{3} p=0 .
$$

All solutions of this equation will tend to zero as $t \rightarrow \infty$ if and only if

$$
A_{1}+A_{2}>0, \quad A_{3}>0,
$$

or equivalently, by (4.12) and $(4.5)_{2}$, if and only if

$$
\frac{\lambda^{\prime}\left(P_{0}\right)}{\lambda\left(P_{0}\right)} \geqq \frac{\beta^{\prime}\left(P_{0}\right)}{\beta\left(P_{0}\right)}-\frac{1}{P_{0}}, \quad \lambda^{\prime}\left(P_{0}\right) \geqq \beta^{\prime}\left(P_{0}\right)
$$

But, by (4.2) and (4.4), (4.15) $)_{2}$ implies $(4.15)_{1}$; thus a necessary and sufficient condition that the equilibrium point $\left(B_{0}, P_{0}\right)$ (and hence also the equilibrium age distribution $\rho_{0}$ ) be asymptotically exponentially stable is that

$$
\lambda^{\prime}\left(P_{0}\right)>\beta^{\prime}\left(P_{0}\right)
$$


In view of $(4.3)_{2}$ and (4.4),

$$
R^{\prime}\left(P_{0}\right)=\frac{1}{\beta\left(P_{0}\right)}\left[\beta^{\prime}\left(P_{0}\right)-\lambda^{\prime}\left(P_{0}\right)\right] .
$$

We therefore conclude from $(4.2)_{2}$ that $(4.16)$ is equivalent to the requirement that

Note that if we let

$$
R^{\prime}\left(P_{0}\right)<0
$$

$$
A=\frac{B}{\beta(P)}
$$

then the system (4.8), (4.9) has the form

$$
\begin{gathered}
\dot{P}+\lambda(P) P-\beta(P) A=0, \\
\dot{A}+\lambda(P) A-[\beta(P)+\alpha] A=0, \\
P(0)=\int_{0}^{\infty} \varphi(a) d a, \quad A(0)=\int_{0}^{\infty} e^{-\alpha a} \varphi(a) d a,
\end{gathered}
$$

which, in applications, may be more amenable to solution.

Now let

$$
\alpha=0 \text {. }
$$

Then (4.20) implies that ${ }^{1} P=A=B / \beta(P)$ with

$$
\begin{gathered}
\dot{P}=\delta(P) P, \\
\delta(P)=\beta(P)-\lambda(P) .
\end{gathered}
$$

When $\delta(P)$ is independent of $P,(4.22)_{1}$ reduces to the Malthusian equation (1.1). On the other hand, when $\delta(P)=\delta_{0}-\omega_{0} P$ with $\delta_{0}>0, \omega_{0}>0,(4.22)_{1}$ yields the Verhulst equation (1.2). It should be noted that even in these simple cases our study provides new information: it enables us to determine the manner in which an initial age distribution $\rho(a, 0)$ evolves in time.

Finally, if we take $\beta(P)=P, \lambda(P)=0$, so that $\delta(P)=P$, then (4.22) 1 reduces to $\dot{P}=P^{2}$. Solutions of this equation become infinite in a finite time. Thus, since this choice of $\beta$ and $\lambda$ conforms to $\left(\mathrm{H}_{1}\right)-\left(\mathrm{H}_{3}\right)$, this example confirms our statement in Section 2 that one cannot expect global existence without additional hypotheses concerning $\beta$ or $\lambda$.

\section{Proofs of Theorems}

Proof of the Lemma. Consider the Banach space $C[0, T]$ with supremum norm $\|\cdot\|_{T}$. Choose $r>0$, let

and let

$$
\Phi=\int_{0}^{\infty} \varphi(a) d a
$$

$$
\Sigma_{T}=\left\{f \mid f \in C^{+}[0, T],\|f-\Phi\|_{T} \leqq r\right\} .
$$

1 This, of course, is consistent with (2.1), (2.2) 2 , and (4.1). 
Since $\Sigma_{T}$ is closed, to complete the proof it suffices to show that $\mathscr{P}_{T}$ maps $\Sigma_{T}$ into itself and is contractive.

Let

$$
\Omega=\{(a, P)|a \geqq 0, P \geqq 0,| P-\Phi \mid \leqq r\} .
$$

Assumption $\left(\mathrm{H}_{2}\right)$ implies that the quantities

$$
\begin{array}{ll}
\lambda_{0}=\sup _{(a, P) \in \Omega} \lambda(a, P), & \lambda_{1}=\sup _{(a, P) \in \Omega} \lambda_{P}(a, P), \\
\beta_{0}=\sup _{(a, P) \in \Omega} \beta(a, P), & \beta_{1}=\sup _{(a, P) \in \Omega} \beta_{P}(a, P)
\end{array}
$$

are all finite For $P \in \Sigma_{T}$ we have then, by $(2.10)_{2},(2.11),(2.13)$, and (5.1),

$$
\mathscr{B}_{T}(P)(t) \leqq \beta_{0} \int_{0}^{t} \mathscr{B}_{T}(P)(a) d a+\beta_{0} \Phi,
$$

and hence Gronwall's inequality implies that

$$
\mathscr{B}_{T}(P)(t) \leqq \beta_{0} \Phi e^{\beta_{0} t} .
$$

Next, we use (2.14), (2.11), (5.1), (5.4), and (5.6) to obtain

for $0 \leqq t \leqq T$ and $P \in \Sigma_{T}$. Since

$$
\begin{aligned}
\left|\mathscr{P}_{T}(P)(t)-\Phi\right| & \leqq \beta_{0} \Phi \int_{0}^{t} e^{\beta_{0} a} d a+\int_{0}^{\infty}|L(a, t ; P)-1| \varphi(a) d a, \\
& \leqq \Phi\left(e^{\beta_{0} T}-1\right)+\Phi \sup _{\substack{a \geqq 0 \\
0 \leqq \tau \leqq T}}|L(a, \tau ; P)-1|,
\end{aligned}
$$

$$
\left|e^{z}-1\right| \leqq|z| e^{|z|}
$$

we conclude, from $(2.11)_{2}$ and (5.4), that

$$
\sup _{\substack{a \geqq 0 \\ 0 \leqq \tau \leqq T}}|L(a, \tau ; P)-1| \leqq \lambda_{0} T e^{\lambda_{0} T} .
$$

The inequalities (5.7) and (5.9) show that $\mathscr{P}_{T}(P) \in \Sigma_{T}$ provided $T$ is sufficiently small. (2.14),

We show next that $\mathscr{P}_{\mathrm{T}}$ is contractive for small $T$. Thus choose $P, \hat{P} \in \Sigma_{T}$. By

$$
\left\|\mathscr{P}_{T}(P)-\mathscr{P}_{T}(\hat{P})\right\|_{T} \leqq \mathrm{I}+\mathrm{II}+\mathrm{III},
$$

where I, II, and III are, respectively, the suprema (over $0 \leqq t \leqq T$ ) of

$$
\begin{aligned}
& \int_{0}^{t}|K(t-a, t ; P)-K(t-a, t ; \hat{P})| \mathscr{B}_{T}(P)(a) d a, \\
& \int_{0}^{t} K(t-a, t ; \hat{P})\left|\mathscr{B}_{T}(P)(a)-\mathscr{B}_{T}(\hat{P})(a)\right| d a \\
& \int_{0}^{\infty}|L(a, t ; P)-L(a, t ; \hat{P})| \varphi(a) d a .
\end{aligned}
$$


We now estimate each of the above. By (2.11), (5.4), and (5.8),

Similarly,

$$
\begin{aligned}
\mid L(a, t ; P)-L(a, t ; \hat{P} \mid & \leqq\left|1-e^{\int_{0}^{j}[\lambda(\tau+a, P(\tau))-\lambda(\tau+a, \hat{P}(\tau))] d a}\right| \\
& \leqq \lambda_{1} T e^{2 \lambda_{0} T}\|P-\hat{P}\|_{T} .
\end{aligned}
$$

$$
\mid K(t-a, t ; P)-K\left(t-a, t ; \hat{P} \mid \leqq \lambda_{1} T e^{2 \lambda_{0} T}\|P-\hat{P}\|_{T} .\right.
$$

From (5.6), (5.12), and (5.13) it follows that for $T$ sufficiently small I and II are each less than $k\|P-\widehat{P}\|_{T}$ with $k \in\left[0, \frac{1}{3}\right)$ a constant (independent of $P$ and $\hat{P}$ ). Thus to complete the proof we have only to show that

for sufficiently small $T$.

$$
\mathrm{II} \leqq \frac{1}{3}\|P-\hat{P}\|_{T}
$$

Let

$$
\zeta(t)=\mathscr{B}_{T}(P)(t)-\mathscr{B}_{T}(\hat{P})(t) .
$$

We then conclude from $(2.10)_{2}$ and the definition of $\mathscr{B}_{T}$ that

$$
\begin{aligned}
\zeta(t)= & \int_{0}^{t} \beta(t-a, P(t)) K(t-a, t ; P) \zeta(a) d a+\int_{0}^{t}[\beta(t-a, P(t)) K(t-a, t ; P) \\
& -\beta(t-a, \hat{P}(t)) K(t-a, t ; \hat{P})] \mathscr{B}_{T}(\hat{P})(a) d a \\
& +\int_{0}^{\infty}[\beta(a+t, P(t)) L(a, t ; P)-\beta(a+t, \hat{P}(t)) L(a, t ; \hat{P})] \varphi(a) d a .
\end{aligned}
$$

Let $f(t)$ denote the last two integrals in (5.16). Then (2.11) and (5.4) imply that

and hence

$$
\zeta(t) \leqq \beta_{0} \int_{0}^{t} \zeta(a) d a+|f(t)|
$$

$$
|\zeta(t) \leqq| f(t)\left|+\beta_{0} \int_{0}^{t}\right| f(a) \mid e^{\beta_{0}(t-a)} d a .
$$

Proceeding as before, we easily verify, with the aid of (2.11), (5.1), (5.4), and (5.6), that

$$
\|f\|_{T} \leqq \kappa_{0}\|P-\hat{P}\|_{T},
$$

where $\kappa_{0}$ is a constant depending only on $\beta_{0}, \beta_{1}, \lambda_{0}, \lambda_{1}$, and $T$. In view of the definition of II, $(2.11)_{1},(5.18)$, and (5.19) imply (5.14) for sufficiently small $T$. This completes the proof.

Proof of Theorem 4. By hypothesis $\left(\mathrm{H}_{1}\right)$ the quantities $\lambda_{0}, \lambda_{1}, \beta_{0}$, and $\beta_{1}$, defined in (5.3), (5.4) are continuous functions of $\Phi$. It is therefore clear from the proof of the lemma that the time-interval $T$ (of that lemma) can be chosen as a continuous function $T(\Phi)$ of $\Phi$. It therefore follows that given any two numbers $0 \leqq \Phi_{0}<\Phi_{1}$ existence is assured for $0 \leqq t \leqq T\left(\Phi_{0}, \Phi_{1}\right)=\min _{\Phi_{0} \leqq \Phi \leqq \Phi_{1}} T(\Phi)$ as 
long as the initial total population $\Phi$ lies in the interval $\Phi_{0} \leqq \Phi \leqq \Phi_{1}$. Choose $\bar{T}>0$. To prove existence for all time, it clearly suffices to establish existence up to time $\bar{T}$. By Theorem 3 , as long as it exists in $0 \leqq t \leqq \bar{T}$ the solution must satisfy $0 \leqq P(t) \leqq \Phi e^{\delta \bar{T}}$. We proceed then on intervals of length $T\left(0, \Phi e^{\delta \bar{T}}\right)$, taking as initial data on each interval the terminal value of $\rho$ on the preceding interval. This obviously leads, in a finite number of steps, to a solution up to time $\bar{T}$.

To prove uniqueness assume that $\rho_{1}$ and $\rho_{2}$ are solutions for all time. Then $\left(B_{1}, P_{1}\right)$ and $\left(B_{2}, P_{2}\right)$ are solutions of $(2.10)$ for all time. By the lemma and the remarks preceding it, we have local uniqueness for the system (2.10). Thus the set of points $t$ such that $\left(B_{1}, P_{1}\right)=\left(B_{2}, P_{2}\right)$ on $[0, t]$ must be an interval of the form $[0, T)$, and, since $B_{k}$ and $P_{k}$ are continuous, $T=\infty$. We therefore conclude from (2.7) and (2.8) that $\rho_{1}=\rho_{2}$. This completes the proof.

Proof of Theorem 5. As we have stated in Section 2, when $\varphi \in C^{1}\left(\mathbb{R}^{+}\right)$, (2.3) is necessary and sufficient for $\rho$ to be continuous. It therefore suffices to show that, granted (2.3), (2.24) is necessary and sufficient for $\rho$ to be of class $C^{\mathbf{1}}$. It follows from our hypotheses and $(2.10)$ that $B, P \in C^{1}\left(\mathbb{R}^{+}\right)$. Thus it is clear from (2.7) and (2.8) that $\rho$ will be of class $C^{1}$ if and only if it has this property across the characteristic $t=a$, or equivalently, if and only if

where

$$
\dot{B}(0)=-\dot{\varphi}(0)-\lambda(0, \Phi) \varphi(0)
$$

$$
\Phi=P(0)=\int_{0}^{\infty} \varphi(a) d a .
$$

The proof of this assertion is greatly facilitated by the change in variable $\tau=t-a+\alpha$ in (2.8). Further, in the derivation of (5.20) we used the relation

$$
\varphi(0)=B(0) \text {, }
$$

which is a direct consequence of (2.3) and (2.10) 2 . Next, by (2.10), (5.21), and (5.22),

$$
\begin{aligned}
& \dot{P}(0)=\dot{\Phi} \\
& \dot{B}(0)=\beta(0, \Phi) \varphi(0)+\int_{0}^{\infty}\left[\beta_{a}(a, \Phi)+\beta_{P}(a, \Phi) \dot{\Phi}-\beta(a, \Phi) \lambda(a, \Phi)\right] \varphi(a) d a
\end{aligned}
$$

where $\dot{\Phi}$ is defined in (2.25). Finally, in view of $(5.23)_{2},(5.20)$ is equivalent to (2.24), and the proof is complete.

Proof of Theorem 7. Let $\rho$ be a solution of the population problem for all time, and let $\xi$ and $p$ be defined by (3.7), so that (3.8) holds. It then follows from $(2.2)_{3}$ that $\xi$ satisfies the initial condition

where

$$
\xi(a, 0)=\eta(a) \quad(a \geqq 0),
$$

$$
\eta(a)=\varphi(a)-\rho_{0}(a)
$$


We begin by obtaining an expression for the "solution" $\xi$ of (3.8) and (5.24) in terms of $x, \psi$, and $\eta$. First of all, proceeding exactly as in the derivation of (2.7) and (2.8), we conclude from (3.8) ${ }_{1}$ that $\xi$ must satisfy the relations

$$
\begin{aligned}
\xi(a, t)= & \eta(a-t) \pi_{0}(a-t, a)+\int_{0}^{t} \pi_{0}(\tau+a-t, a)[x(\tau+a-t, \tau) \\
& -\omega(\tau+a-t) p(\tau)] d \tau \quad \text { for } a \geqq t, \\
\xi(a, t)= & b(t-a) \pi_{0}(a)+\int_{t-a}^{t} \pi_{0}(\tau+a-t, a)[x(\tau+a-t, \tau) \\
& -\omega(\tau+a-t) p(\tau)] d \tau \quad \text { for } a<t
\end{aligned}
$$

where

$$
b(t)=\xi(0, t)
$$

for $t>0$ and

$$
\pi_{0}(a)=e^{-\sum_{0}^{a} \lambda_{0}(a) d a}, \quad \pi_{0}\left(a^{\prime}, a\right)=\frac{\pi_{0}(a)}{\pi_{0}\left(a^{\prime}\right)} .
$$

Further, (5.26)-(5.28) and (3.8) 2,3 imply that

satisfies the matrix equation

$$
\boldsymbol{x}(t)=\left\{\begin{array}{l}
p(t) \\
b(t)
\end{array}\right\}
$$

where

$$
\boldsymbol{A x}(t)+\int_{0}^{t} K(t-\tau) \boldsymbol{x}(\tau) d \tau=f(t)
$$

$$
\begin{gathered}
A=\left\{\begin{array}{rl}
1 & 0 \\
-\kappa & 1
\end{array}\right\}, \quad K=\left\{\begin{array}{ll}
K_{11} & K_{12} \\
K_{21} & K_{22}
\end{array}\right\}, \\
\left\{\begin{array}{l}
K_{11}(t) \\
K_{21}(t)
\end{array}\right\}=\int_{0}^{\infty} \omega(\tau) \pi_{0}(\tau, \tau+t)\left\{\begin{array}{c}
1 \\
-\beta_{0}(\tau+t)
\end{array}\right\} d \tau, \\
\left\{\begin{array}{l}
K_{12}(t) \\
K_{22}(T)
\end{array}\right\}=\pi_{0}(t)\left\{\begin{array}{c}
1 \\
\left.-\beta_{0}(t)\right\}
\end{array}\right\}, \\
f(t)=\int_{0}^{t} \int_{t-\tau}^{\infty} x(\tau+a-t, \tau) \pi_{0}(\tau+a-t, a)\left\{\begin{array}{c}
1 \\
\beta_{0}(a)
\end{array}\right\} d a d \tau \\
+\int_{0}^{\infty} \eta(\tau) \pi_{0}(\tau, \tau+t)\left\{\begin{array}{c}
1 \\
\beta_{0}(\tau+t)
\end{array}\right\} d \tau+\left\{\begin{array}{c}
0 \\
\psi(t)
\end{array}\right\} .
\end{gathered}
$$

It is clear that the properties of the solution $x$ of (5.30) are intimately connected with the properties of the Laplace transform $\hat{K}$ of $K$. By $\left(H_{6}\right),(3.9)_{3}$, and (5.28),

$$
\pi_{0}(a) \leqq e^{-\lambda_{*} a}, \quad \pi_{0}\left(a^{\prime}, a\right) \leqq e^{-\lambda_{*}\left(a-a^{\prime}\right)} \quad\left(a^{\prime} \leqq a\right),
$$

and therefore $(3.9)_{2,6} \cdot\left(\mathrm{H}_{2}\right),\left(\mathrm{H}_{4}\right),(5.28)$, and $(5.31)_{2-4}$ yield the estimate

$$
\left|\frac{d^{k}}{d t^{k}} K(t)\right| \leqq C_{0} e^{-\lambda_{*} t}, \quad k=0,1,2 \text {. }
$$


(Here and in what follows $C_{0}, C_{1}, C_{2}, \ldots$ denote positive constants.) Thus the Laplace transform $\hat{K}(s)$ of $K$ exists for $\operatorname{Re}(s)>-\lambda_{*}$. Let $\Delta(s)$ denote the determinant of the matrix $\boldsymbol{A}+\hat{\boldsymbol{K}}(s)$, so that

$$
\Delta(s)=\left(1+\hat{K}_{11}(s)\right)\left(1+\hat{K}_{22}(s)\right)-\widehat{K}_{12}(s)\left(\hat{K}_{21}(s)-\kappa\right) .
$$

A tedious calculation based on (5.31) $)_{3,4}$ and (3.14) shows that the condition $\Delta(s)=0$ is equivalent to the requirement that (3.15) hold with $\gamma=s$. But our primary hypothesis in Theorem 7 is that (3.15) have no solution $\gamma$ with $\operatorname{Re}(\gamma) \geqq 0$. Thus $\Delta(s)$ has no zeros with $\operatorname{Re}(s) \geqq 0$. Further, it follows from (5.33) and (5.34) that $\Delta(s) \rightarrow 1$ as $s \rightarrow \infty$ with $\operatorname{Re}(s)>-\lambda_{*}$. Thus there exists a positive constant $\bar{\mu}<\lambda_{*}$ such that $\Delta(s)$ has no zeros with $\operatorname{Re}(s) \geqq-\bar{\mu}$, and this, in turn, implies that the matrix $A+\hat{K}(s)$ has an analytic inverse in $\operatorname{Re}(s) \geqq-\bar{\mu}$. Since $\hat{\boldsymbol{K}}(s) \rightarrow 0$ as $s \rightarrow \infty$, this inverse will have the form $A^{-1}+\hat{J}(s)$, where $\hat{J}(s)$ is analytic in $\operatorname{Re}(s) \geqq-\bar{\mu}$ and $\hat{J}(s) \rightarrow 0$ as $s \rightarrow \infty$. In view of (5.33), two integrations by parts yield the estimate

$$
\hat{\boldsymbol{K}}(s)=s^{-1} \boldsymbol{K}(0)+s^{-2} \dot{\boldsymbol{K}}(0)+o\left(s^{-2}\right) \quad \text { as } s \rightarrow \infty .
$$

It follows from (5.35) and the definition of $\hat{J}(s)$ that

$$
\hat{\boldsymbol{J}}(s)=s^{-1} J_{0}+O\left(s^{-2}\right) \quad \text { as } s \rightarrow \infty \text { in } \operatorname{Re}(s) \geqq-\bar{\mu},
$$

where $J_{0}$ is constant, and therefore $\widehat{J}$ is the Laplace transform of the function

$$
J(t)=\frac{1}{2 \pi} e^{-\bar{\mu} t} \int_{-\infty}^{\infty} e^{i \zeta t} \hat{J}(-\bar{\mu}+i \zeta) d \zeta
$$

It follows from (5.37) that

$$
|J(t)| \leqq C_{1} e^{-\bar{\mu} t}
$$

The Laplace transform considerations above indicate that the solution of (5.30) is given by

$$
\boldsymbol{x}(t)=A^{-1} f(t)+\int_{0}^{t} J(t-\tau) f(\tau) d \tau .
$$

(It can be shown that (5.39) does indeed yield the solution of (5.30) whether or not $f(t)$ actually has a Laplace transform.)

Our next step will be to obtain estimates for $|x|=\left(p^{2}+b^{2}\right)^{\frac{1}{2}}$ and $\|\xi(\cdot, t)\|_{L_{1}}$. By the last of (5.31), (5.32), and the fact that $\left\|\beta_{0}\right\|_{L_{\infty}}<\infty$,

$$
|f(t)| \leqq C_{2}\left\{\int_{0}^{t} e^{-\lambda_{*}(t-\tau)}\|x(\cdot, \tau)\|_{L_{1}} d \tau+\|\eta\|_{L_{1}} e^{-\lambda_{*} t}+|\psi(t)|\right\},
$$

and (5.38)-(5.40) imply that

$$
|x(t)| \leqq C_{3}\left\{\|\eta\|_{L_{1}} e^{-\bar{\mu} t}+|\psi(t)|+\int_{0}^{t} e^{-\bar{\mu}(t-\tau)}\left[|\psi(\tau)|+\|\approx(\cdot, \tau)\|_{L_{1}}\right] d \tau\right\}
$$

In deriving (5.41) we have used the following lemma: if $\lambda^{*}>\bar{\mu},|u(t)| \leqq e^{-\bar{\mu} t}$, $|v(t)| \leqq e^{-\lambda_{*} t}$, and $w(t) \geqq 0$, then

$$
|u * v| \leqq C_{4} u, \quad|u * v * w| \leqq C_{5}|u * w|,
$$


where $u * v$ denotes the convolution of $u$ and $v$. Next, since

$$
\int_{0}^{t} \int_{t}^{t} f(a, \tau) d \tau d a+\int_{t}^{\infty} \int_{0}^{t} f(a, \tau) d \tau d a=\int_{0}^{t} \int_{t}^{\infty} f(a, \tau) d a d \tau
$$

we conclude from (5.26), (5.32), (5.41), (5.42), and the inequality $\lambda_{*}>\mu$ that

$$
\begin{aligned}
\|\xi(\cdot, t)\|_{L_{1}} \leqq & \int_{0}^{t} e^{-\lambda_{*} a}|b(t-a)| d a+\|\eta\|_{L_{1}} e^{-\lambda_{*} t} \\
& +\int_{0}^{t} e^{-\lambda_{*}(t-\tau)}\left\{\|x(\cdot, \tau)\|_{L_{1}}+\|\omega\|_{L_{1}}|p(\tau)|\right\} d \tau, \\
\leqq & C_{6}\left\{\|\eta\|_{L_{1}} e^{-\bar{\mu} t}+\int_{0}^{t} e^{-\bar{\mu}(t-\tau)}\left[|\psi(\tau)|+\|x(\cdot, \tau)\|_{L_{1}}\right] d \tau\right\} .
\end{aligned}
$$

Let $\varepsilon$ be a fixed positive number, to be chosen later. By $\left(\mathrm{H}_{5}\right)$ there exists a $\bar{\delta}=\bar{\delta}(\varepsilon)$ such that for $|p|<\bar{\delta}$ we have

$$
|\Lambda(a, p)| \leqq \varepsilon|p|, \quad|\Omega(a, p)| \leqq \varepsilon|p|,
$$

and, without loss in generality, we can assume that $\bar{\delta}<\varepsilon$. Then, since $\left\|\lambda_{0}^{\prime}\right\|_{L_{\infty}}$, $\left\|\beta_{0}^{\prime}\right\|_{L_{\infty}}$, and $\left\|\rho_{0}\right\|_{L_{1}}$ are finite, the last two relations in (3.9) imply that

$$
\|\propto ⿻(\cdot, t)\|_{L_{1}}, \mid \psi(t) \leqq C_{7}\left\{|p(t)|\|\xi(\cdot, t)\|_{L_{1}}+\varepsilon|p(t)|\right\}
$$

with $C_{7}$ independent of $\varepsilon$ provided we require that $\varepsilon<1$. Since $|p|<\bar{\delta}<\varepsilon$, (5.46) yields the inequalities

$$
\|\infty(\cdot, t)\|_{L_{1}},|\psi(t)| \leqq \varepsilon C_{7} \sigma(t), \quad \sigma(t)=\|\xi(\cdot, t)\|_{L_{1}}+|p(t)|
$$

and (5.41), (5.44), and (5.47) imply that

$$
\sigma(t) \leqq C_{8}\left\{\|\eta\|_{L_{1}} e^{-\bar{\mu} t}+\varepsilon \sigma(t)+2 \varepsilon \int_{0}^{t} e^{-\bar{\mu}(t-\tau)} \sigma(\tau) d \tau\right\}
$$

If we require that $\varepsilon<\frac{1}{2 C_{8}}$, then $(5.48)$ implies that

$$
\sigma(t) \leqq K\left\{\|\eta\|_{L_{1}} e^{-\bar{\mu} t}+2 \varepsilon \int_{0}^{t} e^{-\bar{\mu}(t-\tau)} \sigma(\tau) d \tau\right\}
$$

with $K=2 C_{8}$, and Gronwall's inequality yields

$$
\sigma(t) \leqq K\|\eta\|_{L_{1}} e^{(-\bar{\mu}+2 K \varepsilon) t}
$$

We emphasize that this result holds for $0 \leqq t \leqq T$ as long as $|p(t)|<\bar{\delta}$ for $0 \leqq t \leqq T$.

Now choose $\varepsilon>0$ such that $\mu=\bar{\mu}-2 K \varepsilon>0, \varepsilon<1$, and $\varepsilon<\frac{1}{2 C_{8}}$, and then choose $\delta$ such that $\delta<\min (\bar{\delta}(\varepsilon), \bar{\delta}(\varepsilon) / K)$. Assume that the initial data $\varphi$ satisfies $\left\|\varphi-\rho_{0}\right\|_{L_{1}}=\|\eta\|_{L_{1}}<\delta$. Then, since $p(0)=\int_{0}^{\infty} \eta(a) d a$, it follows that $|p(0)|<\delta<\delta$, and we therefore conclude from $(5.47)_{2},(5.50)$, and the continuity of $p$ that $p(t)<\bar{\delta}$ for all $t \geqq 0$. Thus (5.50) is satisfied for all $t \geqq 0$, and, in view of $(3.7)_{2}$ 
and $(5.47)_{2},(3.16)_{1}$ holds. Further, (5.29), (5.41), (5.47), and (5.50) imply that $\|x(\cdot, t)\|_{L_{1}}, \psi(t)$, and $b(t)$ are all $O\left(e^{-\mu t}\right)$, and therefore, since $\|\omega\|_{L_{\infty}}<\infty$, $(5.26)_{2},(5.32)$, and $(3.7)_{1}$ yield $(3.16)_{2}$. This completes the proof.

It is clear from the above proof that, in addition to (3.16), we also have

as $t \rightarrow \infty$.

$$
\left\|\rho(\cdot, t)-\rho_{0}\right\|_{L_{1}}=O\left(e^{-\mu t}\right)
$$

This work was supported by the U.S. National Science Foundation, GURTIN's part under Grant GP 33776X1 and MACCAMY's part under Grant GP 28118.

\section{References}

[1845] VeRHULST, P. F., Recherches mathématiques sur la loi d'accroissement de la population. Mém. Acad. Roy. Bruxelles 18, 1.

[1847] VeRHULST, P. F., Recherches mathématiques sur la loi d'accroissement de la population. Mem. Acad. Roy. Bruxelles 20, 1.

[1925] LotkA, A. J., Elements of Physical Biology. Baltimore: Williams and Wilkins. (Republished as Elements of Mathematical Biology. New York: Dover 1956.)

[1951] Skellam, J. G., Random dispersal in theoretical populations. Biometrika 38, 196-217.

[1954] Andrewartha, H. G., \& L. C. Birch, The Distribution and Abundance of Animals. University of Chicago Press.

[1957] Scherbaum, O., \& G. Rasch, Cell size distribution and single cell growth in Tetrahymena Pyriformis GL. Acta Pathol. Microbiol. Scandinav. 41, 161-182.

[1958] Fisher, R. A., The Genetical Theory of Natural Selection. $2^{\text {nd }}$ ed. New York: Dover.

[1959] KeRNeR, E. H., Further considerations on the statistical mechanics of biological associations. Bull. Math. Biophys. 21, 217-255.

Von FoERSTER, H., Some remarks on changing populations. In The Kinetics of Cellular Proliferation. New York: Grune and Stratton, 382-407.

[1961] LoPeZ, A., Problems in Stable Population Theory. Princeton: Office of Population Research.

[1965] Trucco, E., Mathematical models for cellular systems. The von Foerster equation. Bull. Math. Biophysics 27, 285-305, 449-471.

[1968] Keyfitz, N., Introduction to the Mathematics of Population. Reading: Addison-Wesley.

[1970] Crow, J. F., \& M. Kimura, An Introduction to Population Genetics Theory. New York: Harper and Row.

[1972] LanghaAR, H. L., General population theory in the age-time continuum. J. Franklin Inst. 293, 199-214.

[1973] GurTin, M. E., A system of equations for age-dependent population diffusion. J. Theor. Biol. 40, 389-392. 